\title{
INFLUENCE OF TERFENOL-D POWDER VOLUME FRACTION IN EPOXY MATIRX COMPOSITES ON THEIR MAGNETOMECHANICAL PROPERIES
}

\author{
Rafał MECH*, Jerzy KALETA* \\ *Faculty of Mechanical Engineering, Department of Mechanics, Materials Science and Engineerings, Wrocław University of Technology, \\ Wybrzeze Wyspiańskiego 27, 50-370 Wrocław, Poland \\ rafal.mech@pwr.edu.pl, jerzy.kaleta@pwr.edu.pl
}

received 5 May 2015, revised 22 September 2017, accepted 25 September 2017

\begin{abstract}
In this paper the investigations of magnetostriction as well as DC magnetic properties for composites doped with Terfenol-D particles are presented. All investigations were performed for the materials with $35 \%, 46 \%$ and $70 \%$ volume fraction of the Terfenol-D particles surrounded by epoxy matrix. Moreover, the bulk Terfenol-D alloy was tested. The obtained results show that the magnetization of the composite materials increases with increasing the volume fraction of Terfenol-D particles. Similar dependence as for magnetization was observed for the magnetostriction measurements. Although the magnetostriction of composite material is smaller than for solid Terfenol-D it is still tens of times bigger than in case of traditional magnetostrictive materials. Obtained results gives opportunity to use these materials for variety applications such as actuators and sensors.
\end{abstract}

Key words: Magnetostriction, Magnetic Properties, Smart Materials, Active Materials

\section{INTRODUCTION}

Giant Magnetostrictive Materials (GMM) can convert magnetic energy into mechanical energy (they act as an actuator) or mechanical energy into magnetic energy (sensor) (Engdhal, 2000) with a very high efficiency, which allow them to be widely used in aeronautic, automotive, shipborne and civil engineering constructions as well as in medical sciences, extractive industry, acoustic equipment.

One of the materials which belongs to the Giant Magnetostrictive Materials (GMM) is Terfenol-D (Schwartz, 2002). Due to its unique properties Terfenol-D is widely used in various applications (e.g.: dampers, actuators, harvesters (Arnold, 2007, Daia et al., 2011, Li et al., 2010, Moss et al., 2012, Zhang et al., 2009)). Unfortunately, despite that Terfenol-D has many advantages, the bulk material has also an important drawbacks, such as low tensile strength and the occurrence of eddy currents as a result of work in AC magnetic field at high frequencies (Jia et al., 2009). In order to eliminate such drawbacks researchers work on composites containing the Terfenol-D particles (Kaleta et al., 2011, Dong et al., 2010, Dong et al., 2011, Lu and Li, 2010, Diquet et al., 2009). This involves necessity to examine properties of these new types of composite materials.

In this paper we present the investigation on magnetostriction and DC magnetic properties, for Terfenol-D based composites. The main goal of performed investigations was to determine the role of Terfenol-D particles addition on magnetic properties of the produced materials. Additionally the results obtained for the composites were compared with the bulk Terfenol-D.

\section{MATERIAL}

In this study, the magnetostrictive composites (also referred to as $\mathrm{GMMc}$ ) were used. Samples were made by combining the epoxy resin and the GMM material (Terfenol-D) powder. At first, the epoxy resin Epolam 2015 (from Axons Technologies company) was mixed with the curing agent. Next, the appropriate amount of Terfenol-D powder with particle size of about 5-300 $\mu \mathrm{m}$ (Gansu Tianxing Rare Earth Functional Materials Co., Ltd.) was added. The particles size distribution, and the shape and size of the particles are shown in Fig. 1.

a)

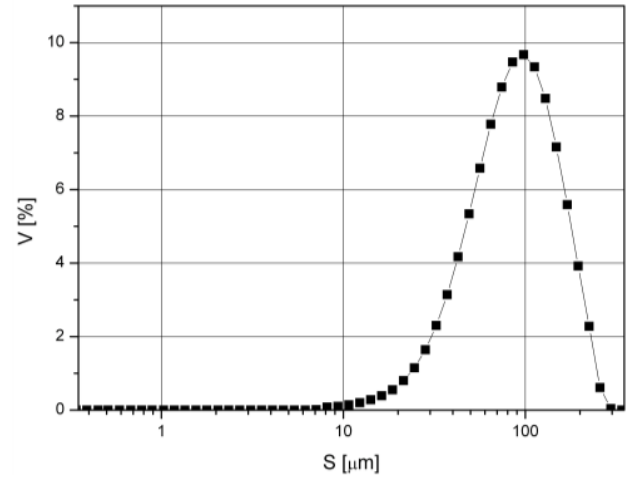

b)

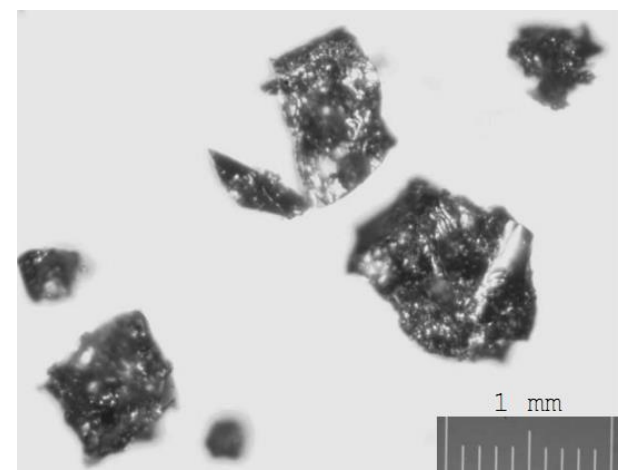

Fig. 1. Terfenol-D powder: a) grain-size analysis, b) the shape and size of the particles 
The manufacturing procedure consisted in intensive mixing of all the ingredients until their complete homogenization. The mixture was then vacuum vented, poured into the dedicated cylindrical containers and subjected to initial polarization, which prevent sedimentation process. After that, the material was once again vented in the vacuum chamber to eliminate the air introduced during the mixing stage. The out-gassed samples were put in between the two strong neodymium magnets (which would lead to their final polarization) until the epoxy resin was completely cured. The scheme of a complete preparation process is shown in Fig. 2. The manufacturing procedure was described in greater detail in (Kaleta et al., 2011).

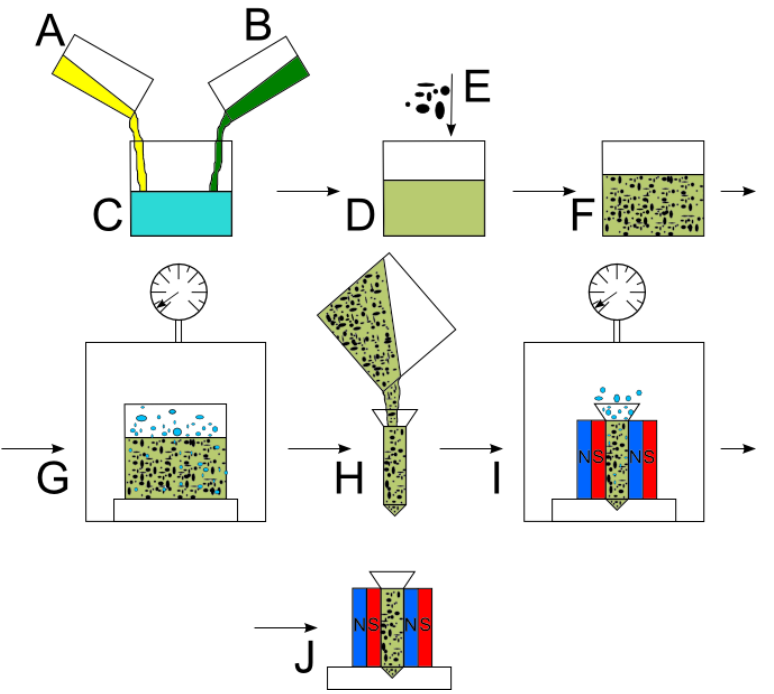

Fig. 2. Scheme of the consecutive processes in the manufacturing of the magnetostrictive composite

The specimens with three $35 \%, 46 \%$ and $70 \%$ volume fraction of Terfenol-D powder were obtained. In short prepared composites were described as composite $35 \%$, composite $46 \%$ and composite $70 \%$. The strong magnetic field $(0,5 \mathrm{~T})$ was used to rotate the particles in such a way that their internal domain structure was parallel to the field lines. As a result of such particle alignment, the magnetic field $(\mathrm{H})$ which, acting parallel to the sample axis, activates the composite, will cause the particles to rotate. In this way the resulting magnetostriction should be stronger than in the case of randomly dispersed particles.

\section{MICROSTRUCTURE}

The goal of investigations was to determine the properties of the produced composite materials and to determine potential differences in relation to the bulk Terfenol-D.

At first observations of a cross-sectional area of the produced composite samples were carried out. These studies were carried out on a scanning electron microscope S-3400N HITACHI. Fig. 3 presents the surface morphology of the composite samples.

More detailed analysis of images with high magnification shows that there is no discontinuity between the applied polymer matrix and Terfenol-D particles. This is mainly due to the low viscosity and relatively low shrinkage of epoxy resin, making it possible to good wetting each of the particles of a magnetostrictive material. a)

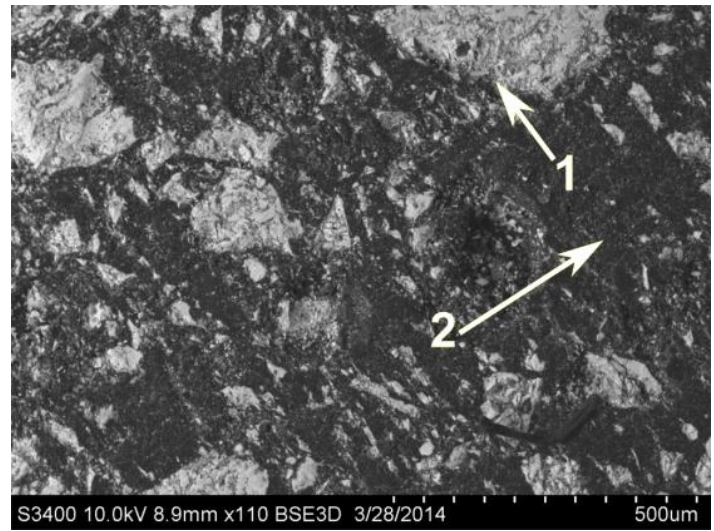

b)

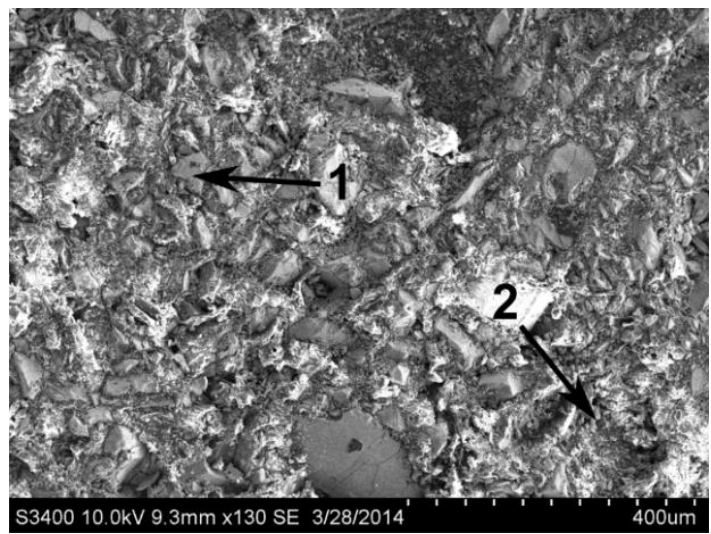

Fig. 3. Image of the composite sample surface made on scanning electron microscope, a) composite $46 \%$ with magnification $110 x$ b) composite $70 \%$ with magnification $130 \mathrm{x}$,

1 - particles of Terfenol-D powder, 2 - epoxy resin

Moreover, studies on the determination of the crystallographic phases of the samples were performed. These studies were carried out in the powder X-ray diffractometer Rigaku Ultima IV.

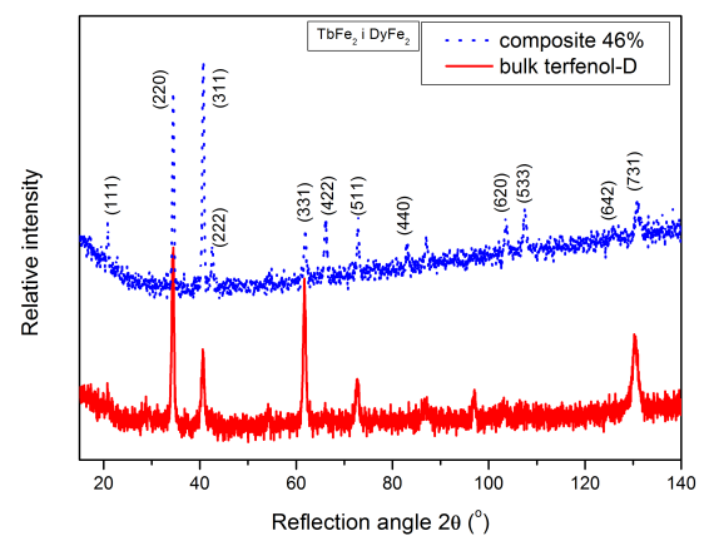

Fig. 4. X-ray diffractograms obtained for the example composite sample (46\%) and bulk Terfenol-D

Obtained results shown in Fig. 4, where the X-ray diffractograms of one of prepared composite sample (composite $46 \%$ ) and bulk Terfenol-D were compared. This measurements allow for the verification of the crystallographic structure of the composite material. The obtained results showed that the methodology of composite material production did not affect in any way on the crystallographic structure of the Terfenol-D particles used during the manufacture of the samples. 


\section{MAGNETOMECHANICAL PROPERTIES}

The tests of magnetomechanical properties were carried out at room temperature, for the specific and constant value of the initial stress which was applied by the MTS testing machine. The machine kept the constant load on each specimen, during the experiment. The magnetic field strength $(\mathrm{H})$ was limited by the magnetic circuit, it was $0 \div 175 \mathrm{kA} / \mathrm{m}$. These measurements were conducted with use of the Hall probe (placed inside the coil), for both positive and negative $\mathrm{H}$ values to check, if the magnetostriction of prepared composite does not depend on the sign of magnetic field. The sample displacement $(\Delta \lambda)$ was measured using the innovative method of Fibre Bragg Grating (FBG) sensors. In this way the influence of the electromagnetic field on the results was eliminated. What is more this is a very precise method of measurements, due to the fact that resolution of the sensor is 2 ppms. The FBG method is described in more detail in (Błażejewski et al., 2011). The strain sensors were placed directly on the specimens. Additionally, during the tests the temperature inside the coil was measured and kept on constant level. All parameters were saved using automatic measurement system.

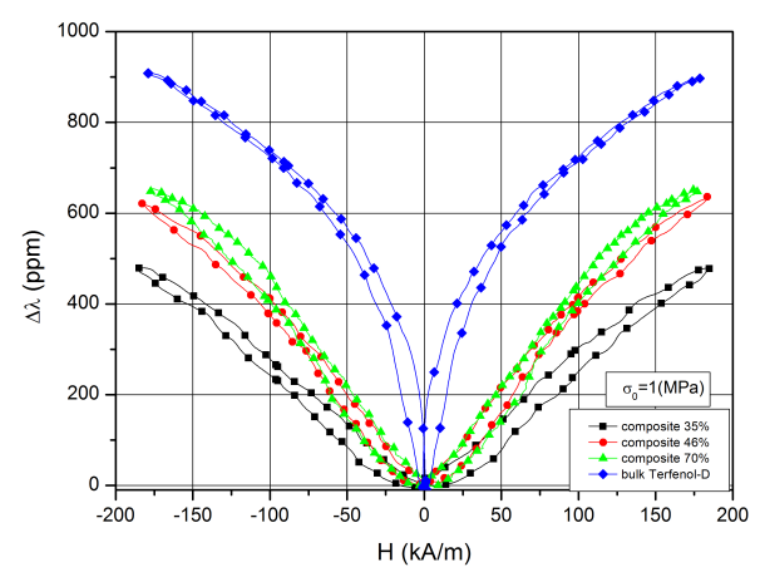

Fig. 5. Magnetostriction $\Delta \lambda$ for the composite material and the monolithic Terfenol-D, at pre-stress $\sigma_{0}=1 \mathrm{MPa}$

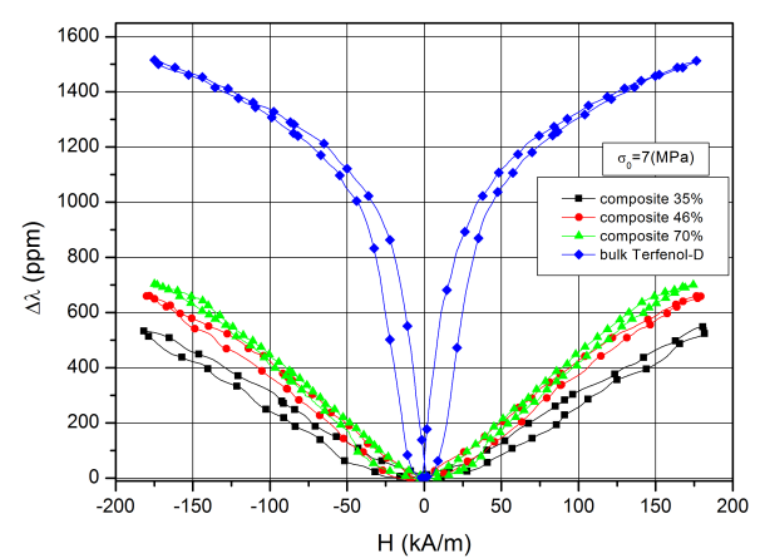

Fig. 6. Magnetostriction $\Delta \lambda$ for the composite material and the monolithic Terfenol-D, at pre-stress $\sigma_{0}=7 \mathrm{MPa}$

The results obtained from the magnetostriction tests performed for the composites and bulk Terfenol-D materials, with prestress of $\sigma_{0}=1 \mathrm{MPa}$ are shown in Fig. 5 . The pre-stress $\sigma_{0}$ is specified as an initial compressive load subjected to the sample during the experiment. It is visible that the maximum value of magnetostriction measured at magnetic field of $175 \mathrm{kA} / \mathrm{m}$ for the composite material consisting 35\% volume fraction of Terfenol-D particles and bulk Terfenol-D alloy is equal to 495ppm and 910ppm, respectively. For the composites with higher volume fraction of Terfenol-D particles the magnetostriction values are higher than for the composite $35 \%$, but it was still lower than for the bulk material. However, the highest value of magnetostriction was obtained for the composite with highest volume fraction of GMM particles (composite 70\%).

a)

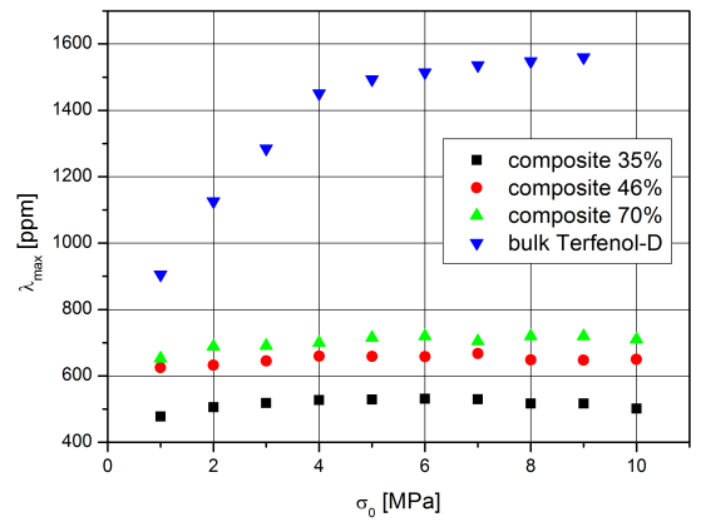

b)

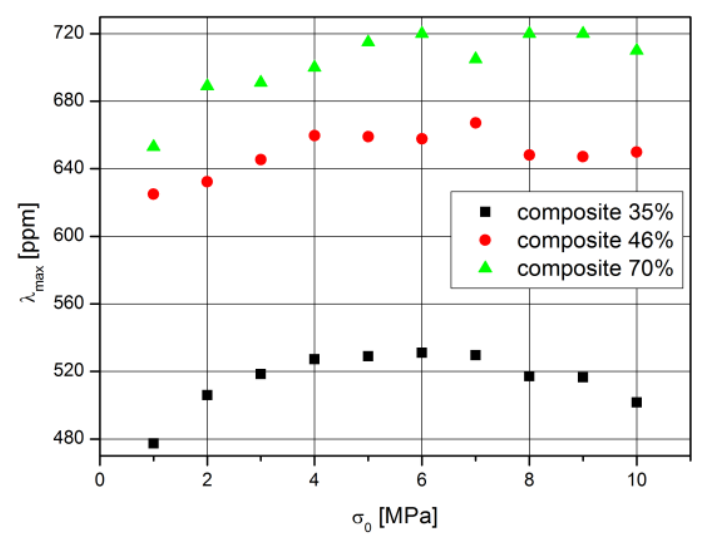

Fig. 7. Influence of the prestress $\sigma_{0}$ on the magnetostriction $\lambda_{\max }$, for a) the composite material and bulk Terfenol-D, and b) for composites materials

In order to determine the magnetomechanical properties of the composites, the magnetostriction values $\Delta \lambda_{\max }$ were compared, i.e. the values obtained for the maximum magnetic field strength $\mathrm{H}=175 \mathrm{kA} / \mathrm{m}$. A comparison of the results for the composite materials and for the bulk Terfenol-D, at $1 \mathrm{MPa}$ of initial stress, is shown in Fig. 5. It is clearly visible that the magnetostriction of composite materials is lower than the magnetostriction of the bulk material. The curves in Fig. 6 presents the comparison of the results obtained for the composite materials and bulk Terfenol-D for the higher value of the pre-stress (7MPa).

Comparing this graph (Fig. 6) with the previous one (Fig. 5) it can be noticed that for the higher pre-stress values the difference in magnetostriction between the composite materials and the bulk Terfenol-D is increasing.

The influence of the change in the pre-stress applied on the composites and the bulk Terfenol-D material, for the whole prestress range, is shown in Fig. 7. It is visible that for composite material the magnetostriction values tend to increase for the prestress up to $6 \mathrm{MPa}$. For the higher values of pre-stress the magnetostriction decrease. There is a similar tendency in the case of the monolithic Terfenol-D, but the point in which the decrease in $\Delta \lambda$ appears is shifted towards higher values. The magnetostriction values attained for the composite are distinctly lower compared to 
the monolithic material. It might be caused by the mutual interactions between the Terfenol-D powder and the composite matrix.

\section{MAGNETIC PROPERTIES}

Magnetic measurements were performed by using a VersaLab unit (Quantum Design) with Vibrating Sample Magnetometer option.

To determine the temperature and DC magnetic field magnetization (M) dependence for the composites and bulk Terfenol-D materials the $M(T, H)$ characteristics were recorded. These investigations were taken in the temperature range from $50 \mathrm{~K}$ to $400 \mathrm{~K}$ and magnetic field range up to $3 \mathrm{~T}$. The exemplary hysteresis loops for the investigated alloys measured at room temperature are shown in Fig. 8.

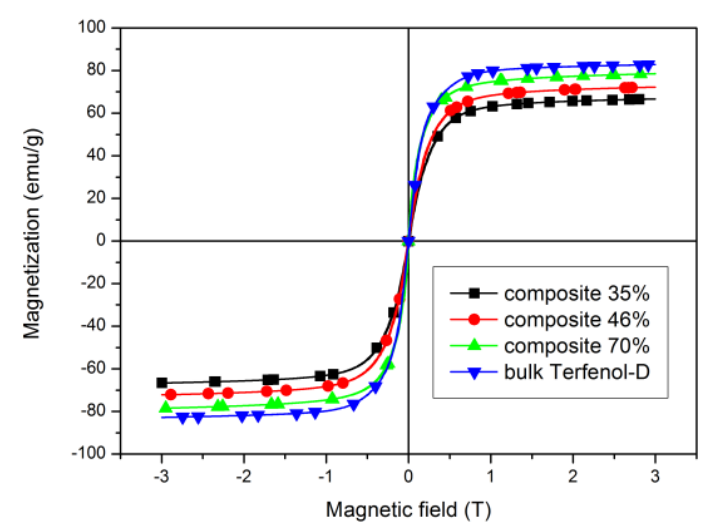

Fig. 8. Room temperature magnetization versus magnetic field for the composites and bulk Terfenol-D material

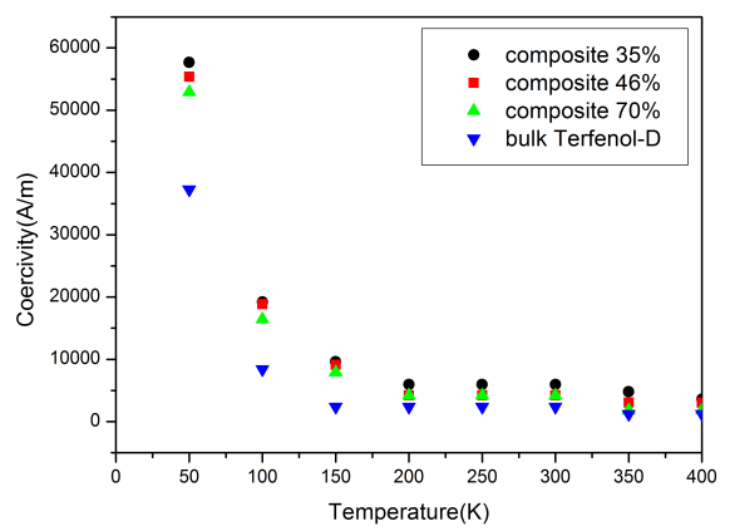

Fig. 9. Coercivity of composite material and solid Terfenol-D as the function of temperature

Changes of coercivity $\mathrm{H}_{\mathrm{c}}$ versus temperature are presented in Fig. 9. The $H_{c}$ value was taken from the magnetization measurements. It is clear that with increasing the measurements temperature decreases value of coercivity. These characteristics are very similar for all tested materials, and are related to the fact that, the magnetic interaction between particles at low temperatures are reduced, and thus the more energy must be provided to rotate the magnetic domains inside of the material. The result of such a characteristic is the appearance of coercive field for measurements of materials at low temperatures. With increasing temperature (greater than 200K) for all examined materials the coercive fields values were reduced.

\section{CONCLUSIONS}

The obtained results show that the magnetization of the composite materials increases with increasing the volume fraction of Terfenol-D particles. The decrease of magnetization with decreasing the volume fraction of Terfenol-D particles in composites is due to the larger distance between the magnetic particles in a polymer resin, and thus decrease in magnetic interactions between them.

Although the magnetostriction of composite material is smaller than for solid Terfenol-D it is still tens of times bigger than in case of traditional magnetostrictive materials such as: iron, nickel, cobalt for which magnetostriction values are respectively 40,50 and 55ppms. Obtained results gives opportunity to use these materials for variety applications such as actuators and sensors. Furthermore in the near future it is possible that these materials will be used also in devices for energy harvesting applications.

\section{REFERENCES}

1. Arnold D.P. (2007), Review of microscale magnetic power generation, IEEE Transactions on Magnetics, 43, 3940-3951.

2. Błażejewski W., Gąsior P., Kaleta J. (2011), Application of Optical Fibre Sensors to Measuring the Mechanical Properties of Composite Materials and Structures, Advances in Composite Materials Ecodesign and Analysis, InTech., 221-246.

3. Daia X., Wena Y., Li P., Yanga J., Li M. (2011), Energy harvesting from mechanical vibrations using multiple magnetostrictive/ piezoelectric composite transducers. Sensors and Actuators A: Physical, 166, 94-101.

4. Diguet G., Beaugnon E., Cavaille J.Y. (2009), From dipolar interactions of a random distribution of ferromagnetic particles to magnetostriction, Journal of Magnetism and Magnetic Materials, 321, 396-401.

5. Dong X., Qi M., Guan X., Ou J. (2010), Microstructure analysis of magnetostrictive composites. Polymer Testing, 29, 369-374.

6. Dong X., Qi M., Guan X., Ou J. (2011), Fabrication of Tbo; $\mathrm{Dy}_{0 ; 7} \mathrm{Fe}_{2} /$ epoxy composites: Enhanced uniform magnetostrictive and mechanical properties using a dry process, Journal of Magnetism and Magnetic Materials, 323, 351-355.

7. Engdhal G. (2000), Handbook of Giant Magnetostrictive Materials, Academic Press.

8. Jia A., Zhang T., Meng H., Jiang C. (2009), Magnetostriction and eddy current loss of bonded giant magnetostrictive particle composites, Acta Metallurgica Sinica, 45(12),1473-1478.

9. Kaleta J., Lewandowski D., Mech R., Zając P. (2011), Metal, ceramic and polymeric composites for various uses., ed. by John Cuppoletti. Rijeka, InTech, cop., 475-504.

10. Li P., Wen Y., Liu P., Li X., Jia C. (2010), A magnetoelectric energy harvester and management circuit for wireless sensor network. Sensors and Actuators A: Physical, 157, 100-106.

11. Lu X.Y., Li H. (2010), Magnetic properties of Terfenol-D film on a compliant substrate, Journal of Magnetism and Magnetic Materials, 322, 2113-2116.

12. Moss S.D., McLeod J.E., Powlesland I.G., Galea S.C. (2012), A biaxial magnetoelectric vibration energy harvester, Sensors and Actuators A: Physical, 175, 165-168.

13. Schwartz M. (2002), Encyclopedia of Smart Materials, John Wiley \& Sons.

14. Zhang C.L., Yang J.S., Chen W.Q. (2009), Harvesting magnetic energy using extensional vibration of laminated magnetoelectric plates, Applied Physics Letters, 95:1, id. 013511 (3 pages).

The work has been accomplished under the grant No. 0401/0029/17 